\title{
Predictable quantum efficient detector for low optical flux measurements
}

\author{
Santeri Porrasmaa ${ }^{1}(1) \cdot$ Timo Dönsberg $^{2} \cdot$ Farshid Manoocheri $^{1} \cdot$ Erkki Ikonen $^{1,2}$ \\ Received: 15 October 2019 / Accepted: 13 January 2020 / Published online: 6 February 2020 \\ (c) The Author(s) 2020
}

\begin{abstract}
The predictable quantum efficient detector (PQED) is a primary standard of optical power, which utilizes two custom-made induced-junction photodiodes that are mounted in a wedged trap configuration for the reduction of reflectance losses. PQED photodiodes of p-type and n-type were characterized for their dark current dependence on reverse bias voltage at room temperature. As simulations predict that the dark current will decrease exponentially with temperature, the temperature dependence of dark current for the n-type photodiodes was also measured. Two n-type induced-junction photodiodes were assembled inside a liquid nitrogen cryostat. The results from the dark current measurements indicate that the cooled n-type photodiodes are suitable for measuring optical fluxes in the few photon regime. A photon flux of approximately 7,000,000 photons per second was measured using the PQED at a cryogenic temperature with a relative standard uncertainty of $0.15 \%$. The results support the utilization of the PQED as a primary standard of optical power in single and few photon applications.
\end{abstract}

Keywords Silicon photodetector · Induced junction · Dark current · Low optical flux

\section{Introduction}

The predictable quantum efficient detector (PQED) is a primary standard of optical power operating in the visible wavelength range [1-3]. The PQED consists of two custommade induced-junction silicon photodiodes [1, 4] arranged in a wedged trap configuration. Over the last decade, the properties of the PQED have been studied comprehensively $[1-3,5]$, and the PQED has been applied in various fields of

Santeri Porrasmaa

santeri.porrasmaa@aalto.fi

Timo Dönsberg

timo.donsberg@vtt.fi

Farshid Manoocheri

farshid.manoocheri@aalto.fi

Erkki Ikonen

erkki.ikonen@aalto.fi

1 Metrology Research Institute, Aalto University, Espoo, Finland

2 National Metrology Institute VTT MIKES, VTT Technical Research Centre of Finland Ltd, Espoo, Finland optical metrology [6-8]. The characterization measurements have shown many appreciable properties, such as highcharge carrier collection efficiency [2,9], low reflectance [1], and linear response over seven orders of magnitude [2]. The uncertainty in the predicted responsivity of the PQED is below 100 parts per million (ppm) [1, 9, 10].

Originally, all PQED photodiodes were based on a structure of thermally grown silicon oxide $\left(\mathrm{SiO}_{2}\right)$ on p-type silicon [1]. Recently, an alternative structure was demonstrated [9], where atomic layer deposition (ALD) was used to grow aluminium oxide $\left(\mathrm{Al}_{2} \mathrm{O}_{3}\right)$ on top of n-type silicon. Two sets of n-type silicon induced-junction photodiodes were manufactured, denoted as set $\mathrm{A}$ and $\mathrm{B}$, with respective doping concentrations of $2.5 \times 10^{11} \mathrm{~cm}^{-3}$ and $4.4 \times 10^{11} \mathrm{~cm}^{-3}$. In addition to smaller doping concentration, set A photodiodes have 16 guard rings, whereas those of set $\mathrm{B}$ have only one. These factors lead to a decrease of dark current by a factor of five at room temperature for the set A photodiodes when compared against set B [9].

Improved dark current characteristics play a major role in low optical flux measurements, as reduced dark current will increase the signal-to-noise ratio (SNR). Additionally, simulations have been carried out to determine dark current 
behaviour when cooling the photodiodes down to cryogenic temperatures $[11,12]$. These simulations indicate that the dark current will decrease exponentially with temperature. Thus, cooling the PQED would drastically increase the dynamic range of the PQED, and would enable the PQED to be used as a primary standard in single and few photon applications [13].

In this study, we present the design of a cryogenic PQED consisting of a liquid nitrogen (LN) cooled cryostat fitted with n-type photodiodes. Dark current dependence on bias voltage at room temperature is presented for both the $\mathrm{n}$ - and p-type PQED photodiodes. The dark current properties of the n-type detector in the temperature range of 76-300 K are also presented. Finally, we demonstrate the possibility of using the PQED as a primary standard in low flux applications.

\section{Cryostat design}

The cryostat and the pumping system used in this study are similar to those described and characterized in [14]. Therefore, only a short description is given here.

The schematic drawing of the cryostat design is shown in Fig. 1. The cryostat is pumped with a turbomolecular pump together with a mechanical backing pump. A radiation shield enclosing the photodiodes is connected directly to a liquid nitrogen (LN) vessel and serves as a cold trap.

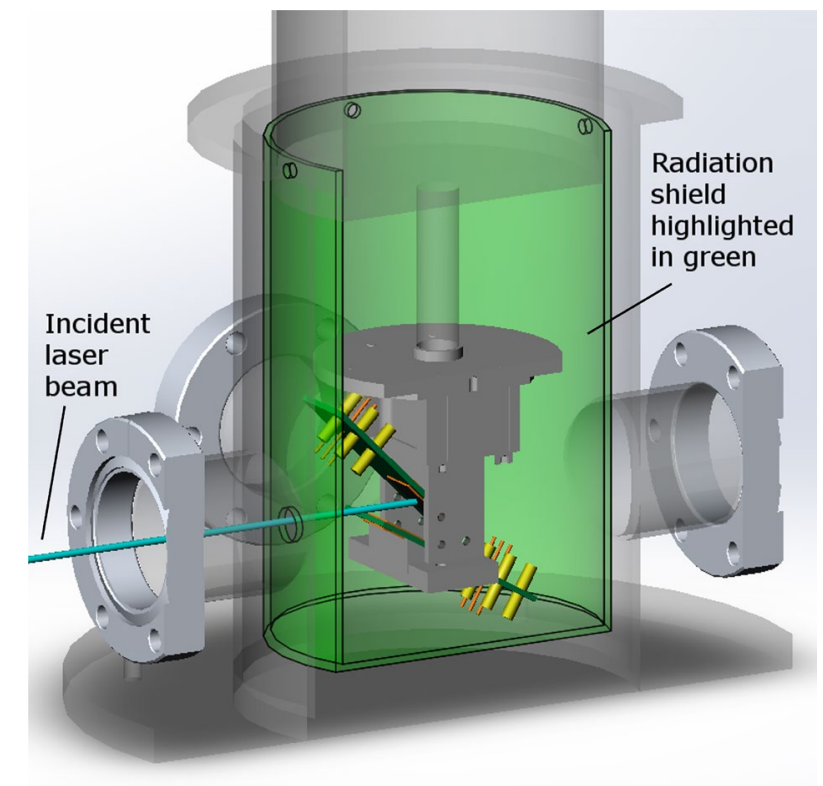

Fig. 1 Schematic drawing of the cryostat. The radiation shield has an aperture of $10 \mathrm{~mm}$ in diameter for the incident laser beam to reach the photodiodes. The central grey part in the figure serves as the photodiode holder. The dimmed grey part above the holder represents a thermal connection between the photodiode holder and the LN vessel
The photodiode holder is in a good copper-to-copper thermal connection with the LN vessel to ensure sufficient heat exchange between them, even under vacuum conditions. The photodiode holder can also be electrically heated. The cryostat is also equipped with a heatable charcoal getter to trap permeated gases. In standard operation, a window aligned in Brewster's angle is placed in front of the photodiodes.

When mounted to the photodiode holder, the PQED photodiodes form a wedged trap configuration (see Fig. 2), similarly to previously characterized PQEDs $[1,3,9]$. The wedged trap configuration and its properties are thoroughly studied in [15], and therefore, only a short description is given. Due to the geometry of the trap structure and the angle of incidence of the incident beam, a total of seven reflections occur in the trap before the back-reflected beam leaves the detector. This structure is calculated to reduce the reflectance significantly [15], and indeed, the measured reflectance values are 20-30 ppm [3] and 100-200 ppm [9], for the p-type and n-type PQEDs, respectively, when using a laser beam at $488.12 \mathrm{~nm}$ wavelength, polarized in the plane of incidence. Although the reflectance of the n-type detector is higher than that of the p-type, the reduced dark current characteristics of the $n$-type detector were considered to be more critical in a few photon applications and were thus chosen for this application.

The cryostat was fitted with two n-type PQED photodiodes from set A [9]. These photodiodes were glued to carrier chips that were manufactured from a silicon wafer. When cooled, this silicon on silicon structure overcomes the problems of previous photodiode designs [1] due to deviating thermal expansion coefficient of the photodiode and the carrier.

To reduce outgassing from the inner surfaces of the cryostat, it was baked-while pumping-at $333 \mathrm{~K}$ for $12 \mathrm{~h}$ before first cooling. When cooled, a vacuum level of around $20 \mu \mathrm{Pa}$ was achieved. Due to the improved vacuum in the cryostat, there is less ice growth on top of the silicon photodiodes $[16,17]$. This in turn means that the temporal change

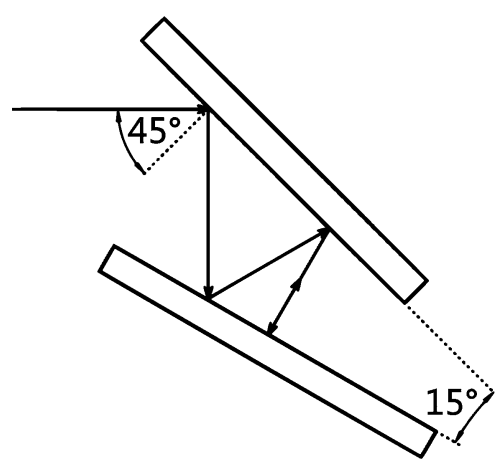

Fig. 2 Schematic drawing illustrating the wedged trap configuration of the photodiodes 
of reflectance observed in [1] is smaller by a factor five, as reported in [14].

\section{PQED dark current}

The temperature dependence of dark current of reverse biased p- and n-type PQED photodiodes has been previously reported in the proximity of room temperature [9]. In this section, the bias voltage dependence of dark current for both types of photodiodes at room temperature and dark current behaviour of the n-type detector when cooled down to $76 \mathrm{~K}$ are studied.

\subsection{Room temperature}

Figure 3 shows the measured dark current of single p-type and n-type PQED photodiodes as a function of bias voltage at $296 \mathrm{~K}$. The photodiodes were thermally isolated from their surroundings to keep the temperature of the photodiodes stable. According to the results, dark current for the n-type photodiode is about $1.3 \mathrm{nA}$ smaller than for the p-type at a reverse bias voltage of $1 \mathrm{~V}$. This difference will further increase with increasing bias voltage, as already demonstrated in [9].

Also shown in Fig. 3 are fitted curves, for which the dark currents $I_{\mathrm{d}}$ are modeled by combining the Shockley diode equation [18] with a resistive leakage term $V_{\mathrm{b}} / R_{1}$ that is assumed to be in parallel with the junction. Thus, the fit equation becomes:

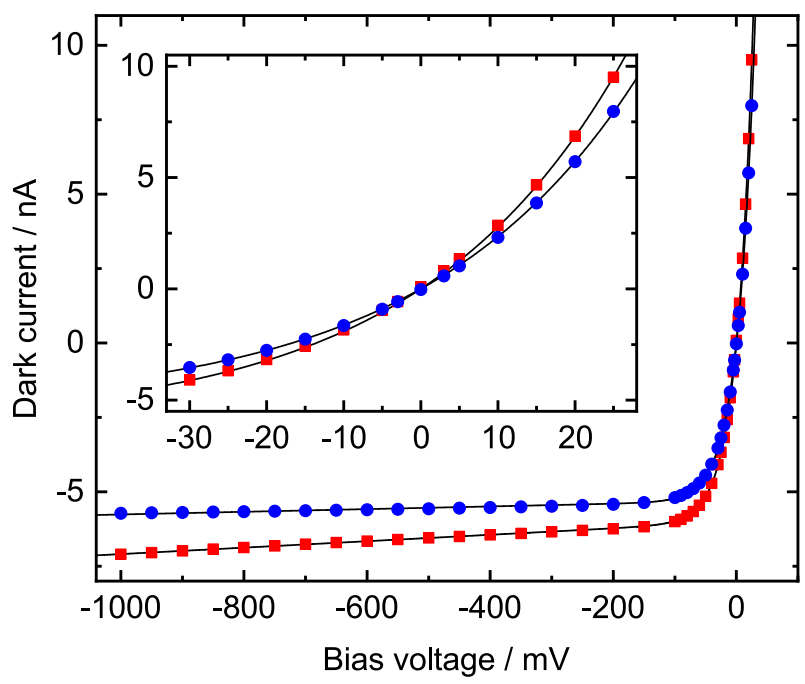

Fig. 3 Measured voltage dependence of dark current for p-type (red squares) and n-type (blue circles) PQED photodiodes at room temperature. The solid lines show the fitted curves
$I_{\mathrm{d}}=I_{\mathrm{s}}\left[\exp \left(\frac{q V_{\mathrm{b}}}{n k T}\right)-1\right]+\frac{V_{\mathrm{b}}}{R_{\mathrm{l}}}$

where $V_{\mathrm{b}}$ is the applied bias voltage, $q$ is the elementary charge, $k$ is the Boltzmann constant, and $T$ is the absolute temperature. The fitted parameters were the saturation current $I_{\mathrm{s}}$, the ideality factor $n$, and leakage resistance $R_{1}$. The ideality factor, which - among other things-accounts for carrier recombination, is equal to one in the ideal case. The obtained values from the fitting are presented for both types of photodiodes in Table 1.

It is noteworthy that the saturation current $I_{\mathrm{s}}$ is highly temperature dependent, and therefore, the obtained fit values are only valid at room temperature. Moreover, Eq. (1) cannot be used to predict the dark current behaviour of the PQED when cooled. However, it indicates that the dark current is governed by a simple system of a near ideal junction and an additional small resistive leakage term. Furthermore, it seems that the higher dark current of the p-type PQED is due to lower parallel leakage resistance rather than higher saturation current of the photodiode junction.

An accurate value for the photodiodes shunt resistance can be calculated by taking the inverse of the fitted function's derivative at $V_{\mathrm{b}}=0 \mathrm{~V}$. The shunt resistance values, also given in Table 1, are obtained using this method. These are of the same order of magnitude as the value of $6 \mathrm{M} \Omega$ estimated in [6].

\subsection{Cooled operation}

To measure the dark current as a function of temperature, the photodiodes were left to warm up from 76 to $300 \mathrm{~K}$ while constantly monitoring the dark current and the photodiode temperature. The photodiodes were nominally reverse biased at $5 \mathrm{~V}$. The results, as shown in Fig. 4, indicate that above $240 \mathrm{~K}$, the dark current decreases roughly by a decade for every decrease of $15 \mathrm{~K}$ in temperature. Below $240 \mathrm{~K}$, the dark current stays fairly constant at around $1 \mathrm{pA}$, and the signal becomes very noisy.

The saturation of dark current when cooled may be explained by an additional leakage component, similar to that seen in the room temperature measurement. It may be that the roughly constant leakage current is produced by the measurement arrangement. Temporal variation of this leakage component would also explain the increased noise.

Table 1 Obtained values from the fitting for saturation current, ideality factor and leakage resistance, and the calculated values of the shunt resistances

\begin{tabular}{lll}
\hline Parameter & n-type & p-type \\
\hline$I_{\mathrm{S}}(\mathrm{nA})$ & 5.31 & 6.01 \\
$n$ & 1.07 & 1.03 \\
$R_{\mathrm{l}}(\mathrm{M} \Omega)$ & 2221.42 & 925.27 \\
$R_{\mathrm{s}}(\mathrm{M} \Omega)$ & 5.14 & 4.37 \\
\hline
\end{tabular}




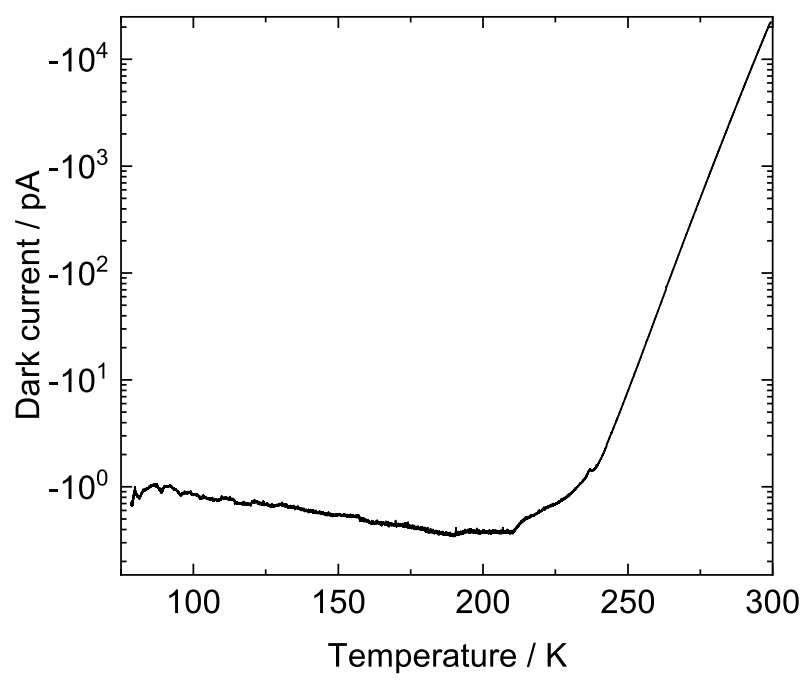

Fig. 4 Temperature dependence of the dark current of two set A photodiodes. Reverse bias of $5 \mathrm{~V}$ was used

\section{Low flux optical power measurement}

The measurements were carried out with an identical setup as described in [9] with the addition of the cryostat on the $X Y$-translation stage and neutral density (ND) filters for attenuating the incident laser beam. For measuring the dark current and low flux photocurrent, the photodiodes were connected to a precision, gain-controllable, commercial transimpedance amplifier (TIA).

Two ND filters with nominal optical densities of 3.0 and 4.0 were used to reduce the power of the stabilized laser beam down to $2.8 \mathrm{pW}$. This corresponds to roughly $7,000,000$ photons per second at a vacuum wavelength of $488.12 \mathrm{~nm}$. While the responsivity simulation of the PQED assumes reverse bias [10], at such a small power level, the uncertainty due to non-biased operation is significantly less than the uncertainty of the measurement [9]. Thus, the photodiodes were not biased. This increases the SNR significantly. In optical power measurement at $76 \mathrm{~K}$ and without biasing, a relative standard uncertainty of $0.15 \%$ was achieved within a 5-min measurement period.

\section{Conclusions}

The dark currents for both p- and n-type PQED photodiodes were characterized for bias voltage dependence at room temperature. As expected, the n-type photodiode exhibits a smaller dark current when compared to the p-type photodiode. A model combining the Shockley diode equation with a parallel resistive leakage term was fitted to the measured values. The obtained fitted values indicate that the higher dark current of the p-type photodiode is due to lower leakage resistance rather than the saturation current of the photodiode junction.

The PQED photodiodes are expected to have exponential decrease in dark current with temperature. Two n-type induced-junction photodiodes housed inside an LN cryostat were characterized for their dark current behaviour when cooled down to LN temperature. It was observed that above $240 \mathrm{~K}$, the dark current decreases roughly by a decade for every decrease of $15 \mathrm{~K}$ in temperature. In our experiment, at temperatures below $240 \mathrm{~K}$, the dark current stayed fairly constant at around $1 \mathrm{pA}$, and the signal became very noisy. It is possible that noise characteristics, and offset currents and voltages of the measurement electronics used in this research affected the signal quality. Further characterization is needed for estimating the effect of measurement electronics on the measured dark current. In addition, other measurement techniques, such as the switched integrator amplifier [19], could be utilized instead of the conventional transimpedance amplifiers.

The n-type photodiodes, cooled down to $76 \mathrm{~K}$, were also used to directly measure the photon flux of roughly $7,000,000$ photons per second at the wavelength of 488.12 $\mathrm{nm}$. The photon flux was obtained by attenuating a stabilized laser beam with two ND filters. With no reverse bias, a relative standard uncertainty of $0.15 \%$ was achieved within a 5-min measurement period. This result indicates that the PQED could be utilized as a primary standard for optical power in the few photon regime. As stated previously, temporally varying leakage current could affect the measurement results. Furthermore, the effect of reverse biasing on responsivity of the detector at low optical fluxes needs to be verified to gain further improvement in the measurement uncertainty.

Acknowledgements Open access funding provided by Aalto University. The work leading to this study is partly funded by the European Metrology Programme for Innovation and Research (EMPIR) Projects 15SIB07 PhotoLED 'Future Photometry Based on Solid State Lighting Products' and 17FUN06 SIQUST 'Single-photon sources as new quantum standards'. The EMPIR initiative is cofunded by the European Union's Horizon 2020 research and innovation programme and the EMPIR Participating States.

Open Access This article is licensed under a Creative Commons Attribution 4.0 International License, which permits use, sharing, adaptation, distribution and reproduction in any medium or format, as long as you give appropriate credit to the original author(s) and the source, provide a link to the Creative Commons licence, and indicate if changes were made. The images or other third party material in this article are included in the article's Creative Commons licence, unless indicated otherwise in a credit line to the material. If material is not included in the article's Creative Commons licence and your intended use is not permitted by statutory regulation or exceeds the permitted use, you will need to obtain permission directly from the copyright holder. To view a copy of this licence, visit http://creativecommons.org/licenses/by/4.0/. 


\section{References}

1. Sildoja, M., Manoocheri, F., Merimaa, M., Ikonen, E., Müller, I., Werner, L., Gran, J., Kübarsepp, T., Smîd, M., Rastello, M.L.: Predictable quantum efficient detector: I. Photodiodes and predicted responsivity. Metrologia 50(4), 385 (2013)

2. Müller, I., Johannsen, U., Linke, U., Socaciu-Siebert, L., Smîd, M., Porrovecchio, G., Sildoja, M., Manoocheri, F., Ikonen, E., Gran, J., et al.: Predictable quantum efficient detector: II. Characterization and confirmed responsivity. Metrologia 50(4), 395 (2013)

3. Dönsberg, T., Sildoja, M., Manoocheri, F., Merimaa, M., Petroff, L., Ikonen, E.: New source and detector technology for the realization of photometric units. Metrologia 51(3), 197 (2014)

4. Hansen, T.: Silicon UV-photodiodes using natural inversion layers. Phys. Scr. 18(6), 471 (1978)

5. Salffner, K., Dönsberg, T., Porrovecchio, G., Smid, M., Nield, K., Nevas, S.: Characterization of a room temperature predictable quantum efficient detector for applications in radiometry and photometry. Metrologia 55(5), 654 (2018)

6. Dönsberg, T., Pulli, T., Poikonen, T., Baumgartner, H., Vaskuri, A., Sildoja, M., Manoocheri, F., Kärhä, P., Ikonen, E.: New source and detector technology for the realization of photometric units. Metrologia 51(6), S276 (2014)

7. Pulli, T., Dönsberg, T., Poikonen, T., Manoocheri, F., Kärhä, P., Ikonen, E.: Advantages of white LED lamps and new detector technology in photometry. Light Sci. Appl. 4(9), 332 (2015)

8. Dönsberg, T., Mäntynen, H., Ikonen, E.: Optical aperture area determination for accurate illuminance and luminous efficacy measurements of LED lamps. Opt. Rev. 23(3), 510 (2016)

9. Dönsberg, T., Manoocheri, F., Sildoja, M., Juntunen, M., Savin, H., Tuovinen, E., Ronkainen, H., Prunnila, M., Merimaa, M., Tang, C.K., et al.: Predictable quantum efficient detector based on n-type silicon photodiodes. Metrologia 54(6), 821 (2017)

10. Gran, J., Kübarsepp, T., Sildoja, M., Manoocheri, F., Ikonen, E., Müller, I.: Simulations of a predictable quantum efficient detector with PC1D. Metrologia 49(2), S130 (2012)
11. Manoocheri, F., Sildoja, M., Dönsberg, T., Merimaa, M., Ikonen, E.: Low-loss photon-to-electron conversion. Opt. Rev. 21(3), 320 (2014)

12. Geist, J., Brida, G., Rastello, M.L.: Prospects for improving the accuracy of silicon photodiode self-calibration with custom cryogenic photodiodes. Metrologia 40(1), S132 (2003)

13. Vaigu, A., Porrovecchio, G., Chu, X.L., Lindner, S., Smid, M., Manninen, A., Becher, C., Sandoghdar, V., Götzinger, S., Ikonen, E.: Experimental demonstration of a predictable single photon source with variable photon flux. Metrologia 54(2), 218 (2017)

14. Manoocheri, F., Dönsberg, T., Sildoja, M., Smíd, M., Porrovecchio, G., Ikonen, E.: Liquid nitrogen cryostat for predictable quantum efficient detectors. J. Phys. Conf. Ser. 972(1), 012021 (2018)

15. Sildoja, M., Manoocheri, F., Ikonen, E.: Reflectance calculations for a predictable quantum efficient detector. Metrologia 46(4), S151 (2009)

16. Berman, A.: Water vapor in vacuum systems. Vacuum 47(4), 327 (1996)

17. Brown, D., George, S., Huang, C., Wong, E., Rider, K.B., Smith, S., Kay, B.D.: $\mathrm{H}_{2} \mathrm{O}$ Condensation coefficient and refractive index for vapor-deposited ice from molecular beam and optical interference measurements. J. Phys. Chem. 100(12), 4988 (1996)

18. Shockley, W.: The theory of $\mathrm{p}-\mathrm{n}$ junctions in semiconductors and p-n junction transistors. Bell Syst. Tech. J. 28(3), 435 (1949)

19. Mountford, J., Porrovecchio, G., Smid, M., Smid, R.: Development of a switched integrator amplifier for high-accuracy optical measurements. Appl. Opt. 47(31), 5821 (2008)

Publisher's Note Springer Nature remains neutral with regard to jurisdictional claims in published maps and institutional affiliations. 